\title{
Haplotype Based Analysis of XRCC3 Gene Polymorphisms in Thyroid Cancer
}

\author{
Romana Sarwar Ishrat Mahjabeen Kashif Bashir Soma Saeed \\ Mahmood Akhtar Kayani
}

Cancer Genetics and Epigenetics Lab, Department of Biosciences, COMSATS Institute of Information

Technology (CIIT), Park Road Chak shazad Islamabad, Pakistan

\author{
Key Words \\ Thyroid cancer • Polymorphisms • DNA repair • Haplotypes
}

\begin{abstract}
Background/Aims: In mammalian cells, XRCC3 plays an important role in the DNA doublestrand breaks (DSBs) repair by homologous recombination. Genetic polymorphisms in XRCC3 gene may potentially affect the repair of DSBs and thus confer susceptibility to thyroid cancer. In this study, we used a haplotype-based approach to investigate whether 5 selected SNPs i.e. rs1799796, rs1799794, rs861539, rs709399 and rs861530 of XRCC3 gene are associated with thyroid cancer risk in 456 cancer patients and 400 cancer-free controls. Methods: Genotyping was performed using Allele-specific PCR followed by sequencing. Statistical analysis was performed to analyse gene and haplotype association. Results: After analysis, frequency of mutant genotype/alleles of SNPs (rs1799796, $p<0.0001 ;$ rs 1799794, $p<0.0001$; rs861539, $p<0.001 ; r s 709399, p<0.0001 ;$ rs861530, $p<0.002$ ) was found significantly higher in thyroid cancer patients compared to controls. Significant associations were found for most of the variant genotypes in SNPs of rs1799794, rs1799796, rs861539, rs861530 and rs709399 in papillary thyroid and follicular cancer patients compared to other histologic subtypes of thyroid carcinoma. Additionally, haplotype analysis revealed that haplotypes, AACGA ( $p=$ $0.0005)$, AGTAA $(p=0.008)$, GATAA $(p=0.001)$, GGCAA $(p=0.001)$ were linked with significant increase in thyroid cancer risk. However, haplotype AGCGG $(p=0.0009)$ was associated with a significant reduced thyroid cancer risk. Conclusion: Our results suggest that common genetic variants in the $X R C C 3$ gene of DSBR pathway may modulate thyroid cancer risk.

\section{Introduction}

(C) 2017 The Author(s)

Published by S. Karger AG, Basel

Thyroid cancer is the most frequent malignancy of thyroid gland and the most increasing endocrine cancer in Pakistan and globally [1]. Its increasing incidence is the main reason for considerable attention of recent researches [2]. In Pakistan, thyroid cancer is responsible for $1.2 \%$ cases of all malignancies, comprising $69-71 \%$ papillary carcinoma, $11-13 \%$ follicular carcinoma, 3-5\% medullary carcinoma and 1-2\% anaplastic carcinoma [3]. Its increasing incidence despite of good survival rate is still alarming in Europe [4, 5], Canada [6, 7], United Kingdom, UK [8] and United States of America, USA [9]. Now it is considered as fastest growing and sixth most common cancer type [10].
\end{abstract}


The DNA double stand breaks (DSBs) are the most lethal type of DNA lesions involved in many tumorigenesis initiation and progression and cause cellular genomic instability [11, 12]. These double strand breaks arise due to exposure to exogenous agents such as ionizing radiations (IRs) and alkylating agents [13]. Homologous recombination repair (HRR) is essentially an error-proof mechanism that occurs during the $S$ or $G_{2}$ phases of the cell cycle, when the sister chromatid can provide a template for accurate repair [14]. Accumulating evidence indicates that a reduced DNA damage repair capacity can lead to a predisposition to DNA damage, mutations and subsequently results in cancer [15]. The precise mechanisms of regulation in the DNA DSBs repair is not well understood, but a number of genes are involved in regulation of DNA repair pathways.

$\mathrm{X}$-ray repair cross-complementing group 3 (XRCC3) is one of the central protein, playing an important role in HRR pathway. XRCC3 interacts and stabilizes RAD51 and involves in HRR for DNA DSBs and cross-link repair in mammalian cells [16]. XRCC3 is a paralog of RAD51, and similar to RAD51, it is essential for genetic stability [17].

Genetic polymorphisms have now been identified in a number of genes involved in repairing DSBs and including genes belonging to the HRR repair pathway. The XRCC3 SNP rs861539 leads to amino acid substitution that may affect the function and/or its interaction with other proteins involved in DNA damage and repair [18]. The SNP rs1799794 (4541 $A>G)$ is located in 5'UTR and the SNP rs1799796 (17893 $A>G)$ is located in exon 5 splice site [19]. Previous studies have examined the relationship between different XRCC3 polymorphisms with the risk of different cancers such as lung cancer, breast cancer, ovarian cancer and head and neck cancer [20-25]. The association between XRCC3 polymorphisms and thyroid cancer risk has not been studied in Pakistani population however, published studies in other populations remain confusing and focussed on single variant rather than haplotype-based gene-gene interaction analysis. Thus we conducted this case-control study to investigate the association of the XRCC3 polymorphism on the risk for thyroid cancer.

\section{Material and Methods}

\section{Study subjects and ethical approval}

The subjects included in this study consisted of 456 diagnosed thyroid cancer patients and 400 age and sex-matched controls. The diagnosis of the thyroid cancer patients were all histologically confirmed at Nuclear medicine department of NORI (Nuclear oncology radiation institute) and PIMS (Pakistan institute of Medical Sciences), Islamabad. Controls were selected from individuals receiving routine medical examinations in these hospitals, and individuals with a previous diagnosis of any type of cancer were excluded. Blood samples from study participants were collected in EDTA vacutainer tubes and a structured questionnaire was filled by interviewing each participant at the time of sample collection about demographic factors, smoking, treatment and dietary habits. This study was approved by the institutional ethical review boards of COMSATS institute of information and technology, Islamabad and both collaborating hospitals, from which patients and controls were recruited. Additionally, all experiments were performed in accordance with relevant guidelines and regulations.

\section{DNA Extraction}

Approximately 3-4 ml blood sample was collected in vacutainer tubes from all enrolled subjects in this study. DNA was extracted by Phenol-Chloroform method with some modifications [26]. The extracted DNA was quantified by $2 \%$ ethidium bromide gels and spectrophotometrically using Nano Drop (Thermoscientific, USA) and stored at $4^{\circ} \mathrm{C}$ until used.

Selection of polymorphisms

The five polymorphisms studied were selected from X-ray repair complementing defective repair in Chinese hamster cells 3 (XRCC3) gene. Selected SNPs included one intronic polymorphism 17893A/G (rs1799796), one 5'UTR splice site polymorphism 4541A/G (rs1799794), one missense mutation in exon 7, 18067C/T or Thr241Met, (rs861539) and two polymorphisms from 3'UTR region 19279C/T (rs709399) 
and 12701G/A (rs861530). The selection of these polymorphisms were based on their wide evaluation in association with different forms of cancer. Primers for PCR amplification were designed by WASP (web based allele specific primer designing tool).

\section{Genotyping}

Selected XRCC3 polymorphisms were genotyped in 456 thyroid cancer patients and 400 controls. ARMS-Polymerase chain reaction was employed to determine the genotypes of all selected hot spot polymorphisms in this study. PCR reaction was carried out in a reaction volume of $10 \mu \mathrm{l}$ containing 50-100ng genomic DNA, $100 \mu \mathrm{M}$ of each primers and Solis BioDyne master mix. The thermal cycling conditions used was: $94^{\circ} \mathrm{C}$ for $1 \mathrm{~min}, 35 \mathrm{cycles}$ of $94^{\circ} \mathrm{C}$ for $30 \mathrm{sec}$, optimized annealing temperature for $45 \mathrm{sec}, 72^{\circ} \mathrm{C}$ for $1 \mathrm{~min}$ and final extension for 7 minutes. The amplified product was visualized on $2 \%$ agarose gel electrophoresis (100V for 45min). ARMS-PCR method was tested with internal control, beta actin as a positive control. PCR products with homozygous wild, homozygous mutant and heterozygous genotype were further confirmed by DNA sequencing analysis and duplicate PCR with random samples.

Statistical analysis

Categorical data were summarized using frequencies and percentiles. Comparison of two groups of categorical variables was performed using Chi- square $(\chi 2)$ test or Fisher's exact test if the number was $<5$. All reported $p$ values were two-sided and $p \leq 0.05$ was considered to be statistically significant. Logistic regression analyses were used to calculate the adjusted odds ratios (ORs) and $95 \%$ confidence intervals (CIs) to determine the risk inflation of different allele/genotype in cancer. Analysis was performed using GraphPad prism software version 6.0.

Haplotype combinations were estimated from gene data. The haplotype analysis and linkage disequilibrium (LD) were performed using Haploview 4.2, in which expectation maximization (EM) algorithm was used to infer haplotypes from the observed genotypes with an unknown linkage phase. The LD coefficient $\left(D^{\prime}\right)<0.2$ was considered to have no $L D, D^{\prime}>0.5$ had a $L D, D^{\prime}>0.8$ had a strong $L D$ and $D^{\prime}=1$ is in complete LD. $r 2$ was the relevant factor. The difference in the distribution of the haplotype frequencies between cases and control groups were assessed with $\chi^{2}$ test. Allele combination analysis was adopted to examine joint effects of five XRCC3 polymorphisms on thyroid cancer risk after adjusting the covariates (age, gender and smoking). Additionally, to address the multiple comparison analysis, a Bonferroni correction was applied to the comparisons. The Bonferroni correction is a conservative method that is free of independency and distribution assumptions. The Bonferroni correction set the significance level at $\alpha$ (alpha) $/ \mathrm{n}$ (number of comparisons). The specific multiple comparisons that were used for each Bonferroni-corrected P value are stated below each table.

\section{Results}

\section{Characteristics of Patients and Controls}

In this study, 456 thyroid cancer patients and 400 control subjects were analyzed for five selected SNPs of XRCC3 gene i.e. rs1799796, rs1799794, rs861539 (Thr241Met), rs709399 and rs861530 and demographic data of these thyroid cancer patients and controls is given in Table 1.

Associations between XRCC3 SNPs and thyroid cancer risk

The genotypes and allele frequencies distribution for five XRCC3 SNPs and thyroid cancer risk are given in Table 2. The homozygous wild-type genotype was used as the reference group to calculate the odd ratios (ORs) and 95\% confidence interval (CI). We found a significant association between XRCC3 SNPs and thyroid cancer risk. For first selected SNP (rs1799794) of XRCC3 gene, frequency of variant G allele (OR: 1.70, 95\% CI: 1.35$2.15, \mathrm{P}<0.0001)$ was significantly higher in patients compared to controls. For the second selected SNP (rs1799796) of XRCC3 gene, the frequency of variant $G$ allele (OR: 1.56, 95\% CI: $1.27-1.91, \mathrm{P}<0.0001$ ) was statistically higher in patients compared to controls as shown in Table 2.

Genotyping of third selected SNP (rs861539) of XRCC3 showed that frequency of homozygous mutant TT (OR: 2.08, 95\% CI: 1.34-3.24, $\mathrm{p}=0.001$ ) along with variant T allele frequen- 
cy (OR: 1.40, 95\% CI: 1.12- Table 1. Frequency distribution analysis of selected demographic $1.75, \mathrm{p}=0.003$ ) were found and risk factors in thyroid cancer cases and controls. ${ }^{\text {FFor }}$ Chi square significantly higher in thyroid test; ${ }^{b}$ Fischer exact test. $\mathrm{OR}=$ odds ratio and $\mathrm{CI}=$ confidence interval. cancer patients group com- ${ }^{*} \mathrm{P} \leq 0.05=$ significant value

pared to controls. For fourth selected SNP (rs861530) of XRCC3 gene, frequencies of the heterozygous mutant (AG) genotype (OR: 2.45 , 95\% CI: 1.33-3.61, $\mathrm{P}=0.0008$ ), homozygous mutant (GG) genotype (OR: 2.39, 95\% CI: 1.52-3.75, $\mathrm{P}=0.002$ ) and variant $\mathrm{G}$ allele frequency (OR: 1.40, 95\% CI: 1.12-1.75, $\mathrm{P}<0.0001)$ were observed statistically significantly higher in patients compared to control group. Genotyping of fifth SNP (rs709399) of XRCC3 gene showed that frequencies of homozygous mutant (AA) genotype (OR: 1.94, 95\% CI: 1.44-2.62, $\mathrm{P}<0.0001$ ) and variant $A$ allele (OR: 2.01, 95\% CI: 1.65-2.44, $\mathrm{P}<0.0001$ ) were significantly associated with increased risk of thyroid cancer compared to controls as shown in Table 2.

Subgroup analysis by different histological types of thyroid cancer was further performed on the association between SNPs and thyroid cancer risk. Significant associations was found for most of the variant genotypes in SNPs of rs1799794, rs1799796, rs861539, rs861530 and rs709399 in papillary thyroid cancer patients. Also significant association existed with follicular thyroid carcinoma in SNPs of rs1799796, rs861539 and rs709399. While only significant association with heterozygous genotype, GA; $\mathrm{p}=0.02$ of rs709399 with medullary thyroid cancer and for heterozygous genotype AG; $p=0.04$ of rs1799796 with anaplastic thyroid cancer was observed as shown in Table 3.

\section{Haplotype analysis of the XRCC3 SNPS}

Haplotype analysis was performed to investigate the effects of all possible haplotypes associated with thyroid cancer disease and to see whether the five SNPs were in linkage disequilibrium. The rare haplotypes (with frequency $<5 \%$ ) were excluded from the associa-

\begin{tabular}{|c|c|c|c|c|}
\hline Genotype / Allele & Cases (456) & Controls (400) & OR $(95 \% \mathrm{CI})$ & ${ }^{*} \mathrm{P}$-value \\
\hline \multicolumn{5}{|c|}{$4541 \mathrm{~A} / \mathrm{G}(\mathrm{rs} 1799794)$} \\
\hline $\mathrm{AA}$ & $289(63 \%)$ & $297(74.3 \%)$ & 1.00 (Ref) & \\
\hline AG & $90(20 \%)$ & $65(16 \%)$ & $1.54(1.06-2.22)$ & 0.02 \\
\hline GG & $77(17 \%)$ & $38(9.7 \%)$ & $1.27(0.82-1.98)$ & 0.27 \\
\hline A allele frequency & $668(73 \%)$ & $659(82 \%)$ & $1.00($ Ref) & 1 \\
\hline G allele frequency & $244(27 \%)$ & $141(18 \%)$ & $1.70(1.35-2.15)$ & $<0.0001$ \\
\hline \multicolumn{5}{|c|}{ 17893A/G(rs1799796) } \\
\hline AA & $284(55.7)$ & $212(53 \%)$ & 1.00 (Ref) & \\
\hline AG & $104(22.8)$ & $128(32 \%)$ & $0.78(1.02-1.84)$ & 0.03 \\
\hline GG & $68(21.5)$ & $60(15 \%)$ & $0.55(1.08-2.20)$ & 0.01 \\
\hline A allele frequency & $672(73)$ & $552(69)$ & $1.00($ Ref $)$ & \\
\hline $\mathrm{G}$ allele frequency & $240(27)$ & $248(37)$ & $0.56(1.27-1.91)$ & $<0.0001$ \\
\hline \multicolumn{5}{|c|}{ 18067C/T or T241M, (rs861539) } \\
\hline $\mathrm{CC}$ & $277(60.8)$ & $273(68.2 \%)$ & 1.00 (Ref) & \\
\hline $\mathrm{CT}$ & $109(23.9)$ & $85(21.2 \%)$ & $1.16(0.84-1.60)$ & 0.35 \\
\hline $\mathrm{TT}$ & $70(15.3)$ & $42(10.5 \%)$ & $2.08(1.34-3.24)$ & 0.001 \\
\hline $\mathrm{C}$ allele frequency & $663(73)$ & $631(79)$ & 1.00 (Ref) & \\
\hline $\mathrm{G}$ allele frequency & 249 (27) & $169(21)$ & $1.40(1.12-1.75)$ & 0.003 \\
\hline \multicolumn{5}{|c|}{ 12701G/A (rs861530) } \\
\hline $\mathrm{AA}$ & $199(44 \%)$ & $222(55.5 \%)$ & 1.00 (Ref) & \\
\hline$A G$ & $150(33 \%)$ & $98(24.5 \%)$ & $2.45(1.33-3.61)$ & 0.0008 \\
\hline GG & $107(23 \%)$ & $80(20 \%)$ & $2.39(1.52-3.75)$ & 0.002 \\
\hline A allele frequency & $548(60 \%)$ & $572(71.5 \%)$ & 1 & \\
\hline $\mathrm{G}$ allele frequency & $364(40 \%)$ & $228(28.5)$ & $1.66(1.36-2.04)$ & $<0.0001$ \\
\hline \multicolumn{5}{|c|}{ 19279G/A (rs709399) } \\
\hline GG & $132(28.9)$ & $198(49.5 \%)$ & 1 & \\
\hline GA & $155(34)$ & $109(27.2 \%)$ & $1.37(1.02-1.84)$ & 0.03 \\
\hline $\mathrm{AA}$ & $169(37.1)$ & $93(23.2 \%)$ & $1.94(1.44-2.62)$ & $<0.0001$ \\
\hline $\mathrm{G}$ allele frequency & $419(46)$ & $505(63)$ & 1 & \\
\hline$A$ allele frequency & $493(54)$ & $295(37)$ & $2.01(1.65-2.44)$ & $<0.0001$ \\
\hline
\end{tabular}
$70(17.5 \%)$ $330(82.5 \%)$ $1.44(1.03-2.02) \quad 0.03^{a}$ $42 \pm 13.2(20-65)$ $179(44.75 \%)$ $221(55.25 \%) \quad 1.03(0.79-1.35) \quad 0.79 a$ $9(2.25 \%)$ $391(97.75 \%)$

4. Smoking History (cigarette, paan, betel quid, moist sniff) $\begin{array}{lllll}\text { Smokers } & 109(24 \%) & 119(30 \%) & 0.55(0.41-0.72) & <0.0001^{\mathrm{a}}\end{array}$ 5. Type of thyroid cancer

Papillary 351(77\%) Follicular $\quad 82(17 \%)$ $\quad 16(3.5 \%)$

Table 2. Genotypic and allelic frequency distribution of XRCC3 SNPs between thyroid cancer cases and controls. Abbreviation: SNP, singe nucleotide polymorphism; OR, odds ratio; CI, confidence interval; P-value calculated by $\chi^{2}$-test 
Sarwar et al.: XRCC3 Polymorphisms and Thyroid Cancer

Table 3. Distribution of genotypes and odds ratios (OR) for different histological subtypes of thyroid carcinoma and controls

\begin{tabular}{|c|c|c|c|c|c|c|c|c|c|c|c|c|c|}
\hline $\begin{array}{l}\text { Genotypes } \\
\text { XRCC3 }\end{array}$ & $\begin{array}{l}\text { Controls } \\
(\mathrm{n}=400)\end{array}$ & $\mathrm{n}$ & $\begin{array}{l}\text { Papillary carcinoma } \\
(\mathrm{n}=351) \\
\text { OR }(95 \% \mathrm{CI})\end{array}$ & ${ }^{*} \mathrm{P}$-value & $\mathrm{n}$ & $\begin{array}{l}\text { Follicular } \\
\text { carcinoma } \\
(\mathrm{n}=82) \\
\text { OR }(95 \% \mathrm{CI})\end{array}$ & ${ }^{*} \mathrm{P}$-value & $\mathrm{n}$ & $\begin{array}{l}\text { Medullary } \\
\text { carcinoma } \\
(\mathrm{n}=16) \\
\text { OR }(95 \% \mathrm{CI})\end{array}$ & ${ }^{*} \mathrm{P}$-value & $\mathrm{n}$ & $\begin{array}{l}\text { Anaplastic } \\
(\mathrm{n}=7) \\
\text { OR }(95 \% \mathrm{CI})\end{array}$ & ${ }^{*} \mathrm{P}$-value \\
\hline \multicolumn{14}{|l|}{ rs1799794 } \\
\hline AA & 297 & 229 & 1.00 (Ref) & & 60 & 1.00 (Ref) & & 9 & 1.00 (Ref) & & 3 & 1.00 (Ref) & \\
\hline $\mathrm{AG}$ & 65 & 79 & $1.49(1.03-2.15)$ & 0.03 & 12 & $0.88(0.45-1.72)$ & 0.71 & 5 & $2.34(0.78-6.96)$ & 0.12 & 2 & $2.06(0.39-10.8)$ & 0.39 \\
\hline $\begin{array}{l}\text { GG } \\
\text { rs1799796 }\end{array}$ & 38 & 47 & $1.47(0.93-2.31)$ & 0.09 & 10 & $1.32(0.63-2.77)$ & 0.45 & 2 & $1.36(0.29-6.21)$ & 0.69 & 2 & $3.81(0.71-20.31)$ & 0.11 \\
\hline $\mathrm{AA}$ & 212 & 133 & 1.00 (Ref) & & 62 & 1.00 (Ref) & & 8 & 1.00 (Ref) & & 1 & 1.00 (Ref) & \\
\hline $\mathrm{AG}$ & 128 & 131 & $1.26(0.93-1.71)$ & 0.12 & 14 & $0.43(0.23-0.80)$ & 0.008 & 4 & $0.70(0.22-2.23)$ & 0.55 & 5 & $5.31(1.01-27.7)$ & 0.04 \\
\hline $\begin{array}{l}\text { GG } \\
\text { rs } 861539\end{array}$ & 60 & 87 & $1.86(1.29-2.69)$ & 0.0008 & 6 & $0.44(0.18-1.07)$ & 0.07 & 4 & $1.88(0.58-6.05)$ & 0.28 & 1 & $0.94(0.11-7.98)$ & 0.95 \\
\hline CC & 273 & 191 & 1.00 (Ref) & & 78 & 1.00 (Ref) & & 6 & 1.00 (Ref) & & 2 & 1.00 (Ref) & \\
\hline СT & 85 & 96 & $1.39(0.99-1.95)$ & 0.05 & 3 & $0.14(0.04-0.45)$ & 0.001 & 6 & $2.22(0.78-6.29)$ & 0.13 & 4 & $4.94(1.08-22.50)$ & 0.03 \\
\hline $\begin{array}{l}\text { TT } \\
\text { rs861530 }\end{array}$ & 42 & 64 & $1.90(1.25-2.89)$ & 0.002 & 1 & $0.10(0.01-0.77)$ & 0.02 & 4 & $2.84(0.87-9.20)$ & 0.08 & 1 & $1.42(0.16-12.08)$ & 0.74 \\
\hline AA & 222 & 147 & 1.00 (Ref) & & 61 & 1.00 (Ref) & & 5 & 1.00 (Ref) & & 1 & 1.00 (Ref) & \\
\hline $\mathrm{AG}$ & 98 & 138 & $1.99(1.46-2.72)$ & $<0.0001$ & 9 & $0.37(0.18-0.78)$ & 0.08 & 7 & $2.39(0.86-6.60)$ & 0.09 & 3 & $2.31(0.50-10.50)$ & 0.27 \\
\hline $\begin{array}{l}\text { GG } \\
\text { rs709399 }\end{array}$ & 80 & 66 & $0.92(0.64-1.33)$ & 0.67 & 12 & $0.68(0.35-1.32)$ & 0.26 & 4 & $1.33(0.41-4.24)$ & 0.62 & 3 & $3.01(0.65-13.6)$ & 0.15 \\
\hline GG & 198 & 51 & 1.00 (Ref) & & 67 & 1.00 (Ref) & & 9 & 1.00 (Ref) & & 5 & 1.00 (Ref) & \\
\hline GA & 109 & 143 & $1.83(1.35-2.49)$ & 0.0001 & 7 & $0.24(0.11-0.55)$ & 0.0007 & 3 & $0.23(0.06-0.83)$ & 0.02 & 2 & $1.06(0.20-5.58)$ & 0.93 \\
\hline $\mathrm{AA}$ & 93 & 157 & $0.93(0.68-1.28)$ & $<0.0001$ & 8 & $0.35(0.16-0.76)$ & 0.008 & 4 & $1.10(0.34-3.49)$ & 0.87 & 0 & $0.21(0.01-3.87)$ & 0.01 \\
\hline
\end{tabular}

tion analyses. The most common haplotypes of the five polymorphisms, calculated by Haploview 4.2 , are summarized in Table 4.

The haplotypes were generated using the five XRCC3 intragenic SNPs, i.e. rs1799796, rs1799794, rs861539 (Thr241Met), rs709399 and rs861530 in the thyroid cancer patients and controls, and 32 different haplotypes were generated which accounted for most of the haplotypes in the case and control group. To decrease the type I error for relatively small sample size, we applied Bonferroni correction and after this correction, only five haplotypes maintained their significance. Among these five haplotypes, AACGA haplotype $(\mathrm{P}=0.0005)$, AGTAA haplotype $\quad(\mathrm{P}=0.008)$, GATAA haplotype $(\mathrm{P}=0.001)$, GGCAA haplotype $(\mathrm{P}=0.001)$ were linked with significant increased risk of thyroid cancer in patient com-
Table 4. Frequency distribution of $X R C C 3$ haplotypes in thyroid cancer cases and controls. Abbreviation: SNP, single nucleotide polymorphism; OR, odds ratio; $\mathrm{CI}$, confidence interval; ${ }^{*} \mathrm{P}$-value calculated by Chi square, $\chi^{2}$-test

\begin{tabular}{|c|c|c|c|c|c|c|c|c|}
\hline \multicolumn{5}{|c|}{ XRCC3 haplotypes (SNPs) } & \multicolumn{2}{|c|}{ Frequency } & \multirow{2}{*}{$x^{2}$} & \multirow[t]{2}{*}{${ }^{*} \mathrm{P}$-value } \\
\hline rs1799794 & rs1799796 & rs861539 & rs709399 & rs861530 & Cases & Controls & & \\
\hline $\mathrm{A}$ & $\mathrm{A}$ & $\mathrm{C}$ & $\mathrm{A}$ & $\mathrm{G}$ & 0.08 & 0.201 & 41.7 & Ref \\
\hline $\mathrm{A}$ & A & $\mathrm{C}$ & A & A & 0.11 & 0.122 & 0.31 & 0.57 \\
\hline $\mathrm{A}$ & A & $\mathrm{c}$ & G & A & 0.07 & 0.033 & 12.0 & 0.0005 \\
\hline $\mathrm{A}$ & A & $\mathrm{C}$ & G & G & 0.06 & 0.095 & 4.80 & 0.02 \\
\hline A & A & $\mathrm{T}$ & A & A & 0.039 & 0.041 & 0.02 & 0.87 \\
\hline $\mathrm{A}$ & A & $\mathrm{T}$ & A & G & 0.037 & 0.060 & 4.48 & 0.03 \\
\hline A & A & $\mathrm{T}$ & G & A & 0.025 & 0.013 & 3.27 & 0.07 \\
\hline $\mathrm{A}$ & A & $\mathrm{T}$ & G & G & 0.025 & 0.021 & 0.34 & 0.56 \\
\hline $\mathrm{A}$ & G & $\mathrm{c}$ & $\mathrm{A}$ & A & 0.067 & 0.043 & 5.15 & 0.02 \\
\hline A & G & $\mathrm{C}$ & A & G & 0.048 & 0.057 & 0.56 & 0.45 \\
\hline A & G & $\mathrm{C}$ & G & A & 0.055 & 0.032 & 5.68 & 0.01 \\
\hline A & G & C & G & G & 0.021 & 0.052 & 10.9 & 0.0009 \\
\hline A & G & $\mathrm{T}$ & A & A & 0.026 & 0.009 & 6.94 & 0.008 \\
\hline A & G & $\mathrm{T}$ & A & G & 0.014 & 0.027 & - & 0.98 \\
\hline A & G & $\mathrm{T}$ & G & A & 0.016 & 0.006 & 3.18 & 0.07 \\
\hline A & G & $\mathrm{T}$ & G & G & 0.038 & 0.010 & 1.12 & 0.28 \\
\hline G & A & C & A & A & 0.022 & 0.037 & 0.03 & 0.86 \\
\hline G & A & C & A & G & 0.024 & 0.038 & 3.29 & 0.06 \\
\hline G & A & C & G & A & 0.020 & 0.010 & 5.29 & 0.02 \\
\hline G & A & C & G & G & 0.015 & 0.024 & 0.16 & 0.68 \\
\hline G & A & $\mathrm{T}$ & A & A & 0.021 & 0.001 & 10.2 & 0.001 \\
\hline G & A & $\mathrm{T}$ & A & G & 0.009 & 0.008 & 2.33 & 0.12 \\
\hline G & A & $\mathrm{T}$ & G & A & 0.014 & 0.001 & - & - \\
\hline G & A & $\mathrm{T}$ & G & G & 0.028 & 0.010 & 0.59 & 0.44 \\
\hline G & G & C & A & A & 0.025 & 0.007 & 0.98 & 0.001 \\
\hline G & G & C & A & G & 0.009 & 0.022 & 0.24 & 0.62 \\
\hline G & G & C & G & A & 0.018 & 0.009 & - & - \\
\hline G & G & C & G & G & 0.009 & 0.006 & 5.44 & 0.01 \\
\hline G & G & $\mathrm{T}$ & A & A & 0.006 & 0.004 & - & - \\
\hline G & G & $\mathrm{T}$ & A & G & 0.004 & 0.000 & - & - \\
\hline G & G & $\mathrm{T}$ & G & A & 0.012 & 0.000 & - & - \\
\hline G & G & $\mathrm{T}$ & G & G & 0.022 & 0.000 & 9.50 & 0.002 \\
\hline
\end{tabular}

Table 5. The distribution of rs1799794 and rs1799796 combined genotypes in thyroid cancer cases and controls. Abbreviations: OR, odds ratios; $\mathrm{CI}$, confidence intervals; $\mathrm{n}$, number of individuals. *P-value calculated by Chi square, $\chi^{2}$-test. $\mathrm{P}$ value after Bonferroni correction i.e. $\mathrm{P}=0.006$ (calculated as $0.05 / 8$ ). $\mathrm{P}$ values in bold have still maintained their significance after Bonferroni correction

\begin{tabular}{ccccc}
\hline rs1799794-rs1799796 & Cases, $\mathrm{n}$ & Controls, $\mathrm{n}$ & OR $(95 \% \mathrm{CI})$ & *P-value \\
\hline AA/AA & 130 & 169 & 1.00 & \\
AA/AG & 102 & 86 & $1.05(0.76-1.45)$ & 0.75 \\
AA/GG & 57 & 42 & $1.21(0.79-1.85)$ & 0.36 \\
AG/AA & 44 & 38 & $1.01(0.64-1.60)$ & 0.94 \\
AG/AG & 24 & 15 & $1.42(0.73-2.75)$ & 0.29 \\
AG/GG & 22 & 12 & $1.64(0.80-3.37)$ & 0.12 \\
GG/AA & 30 & 25 & $1.05(0.61-1.82)$ & 0.84 \\
GG/AG & 28 & 7 & $3.70(1.58-8.50)$ & 0.002 \\
GG/GG & 19 & 6 & $2.85(1.12-7.22)$ & 0.02 \\
\hline
\end{tabular}


pared to controls when AACAG haplotype was taken as reference. However, fifth haplotype AGCGG $(\mathrm{P}=0.0009)$ was associated with a significant reduced risk of thyroid cancer. Following Bonferroni correction, the haplotype analysis did not show any significance for the other haplotypes of XRCC3 gene polymorphisms. Furthermore, no linkage disequilibrium was observed for selected SNPs of XRCC3 gene.

Combined genotype analysis of XRCC3 SNPS

Tables 5-14 summarizes the association studies among the combined genotypes of the five XRCC3 SNPs and overall risk for thyroid cancer. Statistical significant association is considered for each combined genotype after Bonferroni correction. The combined GG/AG genotype frequency (OR: 3.70, 95\% CI: 1.58$8.50, p=0.002$ ) of rs1799794 and rs1799796 maintained its statistical significance to $\sim 4$ fold increase in thyroid cancer risk in patients compared to controls as shown in Table 5. Four fold significant increase in thyroid cancer risk was also observed for rs1799794 and rs861539 in combined AG/ TT genotype (OR: 4.19, 95\% CI: $1.58-11.14, p=0.004)$ in thyroid cancer patients compared to controls (Table 6). For rs1799794rs861530 genotype to genotype interaction, $\sim 5$ fold increased risk was observed in combined GG/AG genotype (OR: 4.77, 95\% CI: 1.81$12.56, \mathrm{p}=0.001$ ) in thyroid cancer patients compared to controls when combined AA/AA wild genotype was taken as reference (Table 7).

The combined AA/AA genotype (OR: 1.64, 95\% CI: 1.17-2.30, $\mathrm{p}=0.003$ ) and GG/GA genotype frequencies (OR: 4.29, 95\% CI: 1.76-10.4, $\mathrm{p}=0.001$ ) for rs1799794 and rs709399 polymorphisms were significantly higher in thyroid cancer patients compared to control group when compared with combined AA/GG genotype as reference. However combined AG/GA $(p=0.03)$ and GG/AA genotype frequencies lost their significant values after Bonferroni correction (Table 8). For rs1799796 and rs861539, combined AG/TT genotype frequency (OR: 2.94, 95\% CI: 1.47-5.86, $\mathrm{p}=0.002$ ) was $\sim 3$ fold higher in thyroid cancer patients versus control group when compared with combined AA/CC wild genotype as reference (Table 9).

Table 6. The distribution of rs1799794 and rs861539 combined genotypes in thyroid cancer cases and controls. Abbreviations: OR, odds ratios; CI, confidence intervals; $n$, number of individuals. *P-value calculated by Chi square, $\chi^{2}$-test. $P$ value after Bonferroni correction i.e. $\mathrm{P}=0.006$ (calculated as $0.05 / 8$ ). $\mathrm{P}$ values in bold have still maintained their significance after Bonferroni correction

\begin{tabular}{lcccc}
\hline rs1799794-rs861539 & Cases & Controls & OR $(95 \% \mathrm{CI})$ & *P-value \\
\hline AA/CC & 178 & 192 & 1.00 & \\
AA/CT & 11 & 71 & $0.72(0.50-1.05)$ & 0.09 \\
AA/TT & 49 & 34 & $1.29(0.81-2.05)$ & 0.26 \\
AG/CC & 53 & 50 & $0.92(0.60-1.39)$ & 0.69 \\
AG/CT & 14 & 10 & $1.23(0.54-2.81)$ & 0.61 \\
AG/TT & 23 & 5 & $4.19(1.58-11.14)$ & 0.004 \\
GG/CC & 46 & 31 & $1.33(0.82-2.15)$ & 0.23 \\
GG/CT & 16 & 3 & $4.00(1.19-10.8)$ & 0.02 \\
GG/TT & 15 & 3 & $4.50(1.29-15.6)$ & 0.01 \\
\hline
\end{tabular}

Table 7. The distribution of rs1799794 and rs861530 combined genotypes in thyroid cancer cases and controls. Abbreviations: OR, odds ratios; CI, confidence intervals; n, number of individuals. *P-value calculated by Chi square, $\chi^{2}$-test. $\mathrm{P}$ value after Bonferroni correction i.e. $\mathrm{P}=0.006$ (calculated as $0.05 / 8$ ). $\mathrm{P}$ values in bold have still maintained their significance after Bonferroni correction

\begin{tabular}{ccccc}
\hline rs1799794-rs861530 & Cases & Controls & OR $(95 \% \mathrm{CI})$ & *P-value \\
\hline AA/AA & 128 & 164 & 1.00 & \\
AA/AG & 90 & 80 & $0.98(0.70-1.37)$ & 0.92 \\
AA/GG & 71 & 53 & $1.20(0.82-1.77)$ & 0.33 \\
AG/AA & 39 & 34 & $1.00(0.62-1.62)$ & 0.97 \\
AG/AG & 34 & 13 & $2.39(1.24-4.61)$ & 0.008 \\
AG/GG & 17 & 18 & $0.82(0.41-1.61)$ & 0.56 \\
GG/AA & 32 & 24 & $1.18(0.68-2.04)$ & 0.54 \\
GG/AG & 26 & 5 & $4.77(1.81-12.56)$ & 0.001 \\
GG/GG & 19 & 9 & $1.88(0.84-4.22)$ & 0.12 \\
\hline
\end{tabular}

Table 8. The distribution of rs1799794 and rs709399 combined genotypes in thyroid cancer cases and controls. Abbreviations: OR, odds ratios; $\mathrm{CI}$, confidence intervals; $\mathrm{n}$, number of individuals. *P-value calculated by Chi square, $\chi^{2}$-test. $P$ value after Bonferroni correction i.e. $\mathrm{P}=0.006$ (calculated as $0.05 / 8$ ). $\mathrm{P}$ values in bold have still maintained their significance after Bonferroni correction

\begin{tabular}{ccccc}
\hline rs1799794-rs709399 & Cases & Controls & OR $(95 \% \mathrm{CI})$ & *P-value \\
\hline AA/GG & 80 & 148 & 1.00 & \\
AA/GA & 94 & 81 & $1.02(0.73-1.42)$ & 0.89 \\
AA/AA & 115 & 68 & $1.64(1.17-2.30)$ & 0.003 \\
AG/GG & 29 & 31 & $0.80(0.47-1.36)$ & 0.42 \\
AG/GA & 33 & 22 & $1.34(0.76-2.33)$ & 0.30 \\
AG/AA & 28 & 12 & $2.11(1.06-4.21)$ & 0.03 \\
GG/GG & 23 & 19 & $1.06(0.57-1.98)$ & 0.84 \\
GG/GA & 28 & 6 & $4.29(1.76-10.4)$ & 0.001 \\
GG/AA & 26 & 13 & $1.80(0.91-3.55)$ & 0.01 \\
\hline
\end{tabular}


No statistical significance was maintained for all combined genotype combinations for rs1799796 and rs861530 compared to combined AA/AA genotype after Bonferroni correction (Table 10). For the rs1799796 and rs709399 combined genotypes distribution, the combined AG/GG genotype (OR: 2.05 , 95\% CI: 1.26-3.33, $\mathrm{p}=0.003$ ) and combined GG/AA genotype frequencies (OR: 3.02, 95\% CI: $1.59-5.71, p=0.0007$ ) were significantly higher in patients compared to controls. The combined AA/GG wild genotypes were used as reference (Table 11). For rs861539 and rs861530 combined genotype distribution, only combined TT/GG genotype frequency (OR: 3.16, 95\% CI: 1.42-7.03, $\mathrm{p}=0.004$ ) increased $\sim 4$ fold risk in thyroid cancer patients versus controls when compared with combined CC/GG genotype (Table 12). For rs861539 and rs709399 combined genotype analysis when compared with wild combined CC/ GG genotype as reference, combined CC/AA genotype (OR: 1.61, 95\% CI: $1.15-2.27, \mathrm{p}=0.005)$ and combined TT/AA genotype frequency (OR: 3.39, 95\% CI: $1.45-7.90, p=0.004$ ) were significantly higher in thyroid cancer patients compared to controls as shown in Table 13. After Bonferroni correction, for combined genotype analysis of rs861530 and rs709399, only combined AG/AA genotype frequency maintained its significance and $\sim 3$ fold increased risk of thyroid cancer was observed in patients compared to controls when combined AA/GG genotype was taken as reference for comparison (Table 14).

\section{Discussion}

Targeting DNA damage becomes an effective approach to increase the efficiency of radiation therapy [27]. X-ray repair complementing defective repair in Chinese hamster cells 3 (XRCC3) belongs to a family of genes responsible for repairing DNA double strand breaks caused by normal metabolic processes or exposure to ionizing radiation [20]. XRCC3 proteins are recruited to a DSB during the S and G2/M phase of the cell cycle. Considering the importance of XRCC3 to maintain chromosomal stability by repairing most lethal DSBs and inhibiting tumorigenesis, we hypothesized that change in XRCC3 expression affected by
Table 9. The distribution of rs1799796 and rs861539 combined genotypes in thyroid cancer cases and controls. Abbreviations: OR, odds ratios; $\mathrm{CI}$, confidence intervals; $\mathrm{n}$, number of individuals. ${ }^{*} \mathrm{P}$-value calculated by Chi square, $\chi^{2}$ -test. $\mathrm{P}$ value after Bonferroni correction i.e. $\mathrm{P}=0.006$ (calculated as $0.05 / 8$ ). P values in bold have still maintained their significance after Bonferroni correction

\begin{tabular}{ccccc}
\hline rs1799796-rs861539 & Cases & Controls & OR (95\% CI) & *P-value \\
\hline AA/CC & 122 & 153 & 1.00 & \\
AA/CT & 48 & 53 & $0.77(0.50-1.16)$ & 0.21 \\
AA/TT & 34 & 26 & $1.15(0.68-1.96)$ & 0.58 \\
AG/CC & 94 & 79 & $1.05(0.74-1.47)$ & 0.75 \\
AG/CT & 25 & 18 & $1.23(0.66-2.29)$ & 0.51 \\
AG/TT & 35 & 11 & $2.94(1.47-5.86)$ & 0.002 \\
GG/CC & 61 & 41 & $1.35(0.88-2.05)$ & 0.15 \\
GG/CT & 19 & 14 & $1.19(0.59-2.42)$ & 0.61 \\
GG/TT & 18 & 5 & $3.24(1.19-8.82)$ & 0.02 \\
\hline
\end{tabular}

Table 10. The distribution of rs1799796vs rs861530 combined genotypes in thyroid cancer cases and controls. Abbreviations: OR, odds ratios; $\mathrm{CI}$, confidence intervals; n, number of individuals. *P-value calculated by Chi square, $\chi^{2}$-test. $\mathrm{P}$ value after Bonferroni correction i.e. $\mathrm{P}=0.006$ (calculated as $0.05 / 8$ ). $\mathrm{P}$ values in bold have still maintained their significance after Bonferroni correction

\begin{tabular}{ccccc}
\hline rs1799796- rs861530 & Cases & Controls & OR $(95 \% \mathrm{CI})$ & *P-value \\
\hline $\mathrm{AA} / \mathrm{AA}$ & 90 & 141 & 1.00 & \\
$\mathrm{AA} / \mathrm{AG}$ & 65 & 46 & $1.27(0.85-1.91)$ & 0.23 \\
$\mathrm{AA} / \mathrm{GG}$ & 49 & 45 & $0.94(0.61-1.45)$ & 0.81 \\
$\mathrm{AG} / \mathrm{AA}$ & 63 & 59 & $0.92(0.63-1.35)$ & 0.69 \\
$\mathrm{AG} / \mathrm{AG}$ & 53 & 29 & $1.68(1.04-2.70)$ & 0.03 \\
$\mathrm{AG} / \mathrm{GG}$ & 38 & 20 & $1.72(0.98-3.02)$ & 0.05 \\
GG/AA & 46 & 22 & $1.92(1.13-3.26)$ & 0.01 \\
GG/AG & 32 & 23 & $1.23(0.71-2.15)$ & 0.45 \\
GG/GG & 20 & 15 & $1.17(0.59-2.33)$ & 0.63 \\
\hline
\end{tabular}

Table 11. The distribution of rs1799796 and rs709399 combined genotypes in thyroid cancer cases and controls. Abbreviations: OR, odds ratios; $\mathrm{CI}$, confidence intervals; $\mathrm{n}$, number of individuals. ${ }^{*}$ P-value calculated by Chi square, $\chi^{2}$ -test. $\mathrm{P}$ value after Bonferroni correction i.e. $\mathrm{P}=0.006$ (calculated as $0.05 / 8)$. P values in bold have still maintained their significance after Bonferroni correction

\begin{tabular}{ccccc}
\hline rs1799796-rs709399 & Cases & Controls & ORs $(95 \%$ CI $)$ & *P-value \\
\hline AA/GG & 54 & 124 & 1.00 & \\
AA/GA & 80 & 54 & $1.36(0.93-1.98)$ & 0.10 \\
AA/AA & 70 & 54 & $1.16(0.79-1.70)$ & 0.44 \\
AG/GG & 49 & 43 & $0.99(0.64-1.54)$ & 0.99 \\
AG/GA & 48 & 39 & $1.08(0.69-1.70)$ & 0.70 \\
AG/AA & 57 & 26 & $2.05(1.26-3.33)$ & 0.003 \\
GG/GG & 29 & 31 & $0.80(0.47-1.36)$ & 0.42 \\
GG/GA & 27 & 16 & $1.51(0.80-2.84)$ & 0.20 \\
GG/AA & 42 & 13 & $3.02(1.59-5.71)$ & 0.0007 \\
\hline
\end{tabular}


polymorphisms in promoter, exonic and intronic region might modulate the pathogenesis of thyroid cancer. Present study was designed to assess the association of five selected SNPs with thyroid carcinogenesis. We also investigated whether or not the five SNPs were in linkage disequilibrium or association of common haplotypes of these SNPs with thyroid carcinogenesis. Additionally, we also estimated the association among the combined genotypes of these SNPs and overall risk of thyroid cancer.

In this study, the variant allele frequencies for selected XRCC3 polymorphisms (rs1799794, rs861539, rs861530 and rs709399) were found to increase the risk of thyroid cancer, however GG genotype frequency of rs1799796 was found to decrease the thyroid cancer risk. First two SNP rs1799794, rs861530 included in this study are located in $3^{\prime}$ UTR region of XRCC3 and their functional significance has not yet been elucidated. However, their $3^{\prime}$ UTR location can affect polyadenylation and alterations in regulatory protein-mRNA and miRNA-mRNA interactions. It can cause disease directly, modifying the severity of the disease phenotype, or be linked with disease susceptibility [28-30]. Third SNP with increased risk of thyroid cancer, rs861539 (Thr241 Met in exon 5 coding region) is the most extensively studied genetic variant of XRCC3 [31-33] and evidence indicates that the Thr/Met variant resides in the adenosine triphosphate-binding domain of $X R C C 3$, the only domain with known functional activity [34]. This fact suggests that the change caused by the XRCC3 Thr241Met polymorphism is associated with other proteins, especially $R A D 51$ [35]. The product of XRCC3 is a RAD51 paralog necessary for the binding and stabilization of the latter during DNA repair. In our study, the increased risk of rs861539 polymorphism in thyroid cancer is comparable to previous results reporting significant associations with thyroid cancer risk [36-40]. Nevertheless, many studies have found no evident correlation between XRCC3 polymorphisms and thyroid cancer $[41,42]$. The rs861539 polymorphism has also been reported associated with other cancers such as colorectal cancer [43, 44], ovarian cancer [19], glioma [45], bladder cancer $[32,46]$ and acute myeloid leukemia $[47,48]$.

Table 12. The distribution of rs861539 and rs861530 combined genotypes in thyroid cancer cases and controls. Abbreviations: OR, odds ratios; $\mathrm{CI}$, confidence intervals; $\mathrm{n}$, number of individuals. ${ }^{*}$ P-value calculated by Chi square, $\chi^{2}$ -test. $\mathrm{P}$ value after Bonferroni correction i.e. $\mathrm{P}=0.006$ (calculated as $0.05 / 8)$. P values in bold have still maintained their significance after Bonferroni correction

\begin{tabular}{ccccc}
\hline rs861539-rs861530 & Cases & Controls & OR $(95 \% \mathrm{CI})$ & *P-value \\
\hline CC/AA & 121 & 147 & 1.00 & \\
CC/AG & 93 & 68 & $1.25(0.88-1.76)$ & 0.20 \\
CC/GG & 63 & 58 & $0.94(0.64-1.38)$ & 0.77 \\
CT/AA & 38 & 54 & $0.58(0.37-0.90)$ & 0.01 \\
CT/AG & 33 & 17 & $1.75(0.96-3.20)$ & 0.06 \\
CT/GG & 21 & 14 & $1.33(0.66-2.65)$ & 0.41 \\
TT/AA & 40 & 21 & $1.78(1.00-2.99)$ & 0.04 \\
TT/AG & 24 & 13 & $1.65(0.83-3.29)$ & 0.15 \\
TT/GG & 23 & 8 & $3.16(1.42-7.03)$ & 0.004 \\
\hline
\end{tabular}

Table 13. The distribution of rs861539vs rs709399 combined genotypes in thyroid cancer cases and controls. Abbreviations: OR, odds ratios; $\mathrm{CI}$, confidence intervals; $\mathrm{n}$, number of individuals. ${ }^{*} \mathrm{P}$-value calculated by Chi square, $\chi^{2}$ -test. $\mathrm{P}$ value after Bonferroni correction i.e. $\mathrm{P}=0.006$ (calculated as $0.05 / 8$ ). $\mathrm{P}$ values in bold have still maintained their significance after Bonferroni correction

\begin{tabular}{ccccc}
\hline rs861539-rs709399 & Cases & Controls & OR $(95 \% \mathrm{CI})$ & *P-value \\
\hline CC/GG & 74 & 134 & 1.00 & \\
CC/GA & 94 & 74 & $1.14(0.81-1.60)$ & 0.43 \\
CC/AA & 109 & 65 & $1.61(1.15-2.27)$ & 0.005 \\
CT/GG & 25 & 45 & $0.45(0.27-0.76)$ & 0.002 \\
CT/GA & 33 & 19 & $1.56(0.87-2.79)$ & 0.13 \\
CT/AA & 34 & 21 & $1.45(0.82-2.54)$ & 0.19 \\
TT/GG & 33 & 19 & $1.56(0.87-2.79)$ & 0.13 \\
TT/GA & 28 & 16 & $2.941 .57(0.83-)$ & 0.16 \\
TT/AA & 26 & 7 & $3.39(1.45-7.90)$ & 0.004 \\
\hline
\end{tabular}

Table 14. The distribution of rs861530 and rs709399 combined genotypes in thyroid cancer cases and controls. Abbreviations: OR, odds ratios; $\mathrm{CI}$, confidence intervals; $\mathrm{n}$, number of individuals. ${ }^{*} \mathrm{P}$-value calculated by Chi square, $\chi^{2}$ -test. $\mathrm{P}$ value after Bonferroni correction i.e. $\mathrm{P}=0.006$ (calculated as $0.05 / 8$ ). P values in bold have still maintained their significance after Bonferroni correction

\begin{tabular}{ccccc}
\hline rs861530-rs709399 & Cases & Controls & OR $(95 \% \mathrm{CI})$ & *P-value \\
\hline $\mathrm{AA} / \mathrm{GG}$ & 49 & 100 & 1.00 & \\
$\mathrm{AA} / \mathrm{GA}$ & 75 & 64 & $1.03(0.71-1.48)$ & 0.85 \\
$\mathrm{AA} / \mathrm{AA}$ & 75 & 58 & $1.16(0.79-1.68)$ & 0.43 \\
$\mathrm{AG} / \mathrm{GG}$ & 54 & 56 & $1.03(0.71-1.48)$ & 0.85 \\
$\mathrm{AG} / \mathrm{GA}$ & 41 & 25 & $1.48(0.88-2.48)$ & 0.13 \\
$\mathrm{AG} / \mathrm{AA}$ & 55 & 17 & $3.09(1.76-5.41)$ & 0.0001 \\
GG/GG & 29 & 42 & $0.57(0.35-0.94)$ & 0.03 \\
GG/GA & 39 & 20 & $1.77(1.01-3.10)$ & 0.04 \\
GG/AA & 39 & 18 & $2.00(1.11-3.52)$ & 0.01 \\
\hline
\end{tabular}


Fourth studied SNP of XRCC3, rs1799794, is located in the 5' UTR region and significant association of rs1799794 was observed with increased risk of thyroid cancer in this study. The underlying mechanism of its function may be related to its alteration in local DNA secondary structure or functional motif, thereby affecting the binding affinities of the relevant transcription factors [49]. In earlier studies, rs1799794 has been observed associated with increased risk of overall cancer [50], breast cancer [23], bladder cancer [51, 52]. Nevertheless, decreased risk of ovarian cancer has also been observed in some of the studies [20].

The results of haplotype analysis were consistent with the results of genotypic analysis, carriers of XRCC3 haplotypes containing one of high risk alleles AACGA, AGTAA, GATAA and GGCAA had a significantly increased risk of thyroid cancer compared with those of the common haplotype AACAG. However haplotype AGCGG was associated with decreased risk of thyroid cancer. The possible reason for this protective role may be due to presence of $\mathrm{G}$ allele of rs1799796. It has earlier been reported that rs1799794/rs1799796/rs861539 haplotypes may decrease the risk of thyroid cancer and behave as a protective factor [53]. In one of the earlier haplotype based case-control study in hepatocellular carcinoma (HCC) risk in Chinese population, concluded that carriers with T allele of rs861539, G allele of rs861530 and rs3212092 T allele (TTG haplotype) have increased risk of HCC [54] which is comparable to our results. Two previous studies of haplotypes inferred from three XRCC3 SNPs (rs1799794/rs1799796/rs861539) concluded that breast cancer risk is increased among individuals with "GAT" haplotype [55, 56]. It has been observed that XRCC3 241Met allele and its associated haplotype (4552A/18067T) were significantly inversely associated risk of squamous and basal cell carcinoma [57]. Smedby et al. [58] showed that the XRCC3 haplotype-TCGTAAC (rs32120 24/rs3212038/rs3212057/ rs3212068/rs3212090) was more common for follicular lymphoma cases than controls. Finally, Zhou et al. [45] reported that rs861530 G and $3212092 \mathrm{~T}$ high-risk alleles contributed independently to risk of gliomas.

Our results of SNP-SNP interaction of XRCC3 showed that the twelve combinations (rs1799794-GG with rs1799796-AG, rs1799794-AG with rs861539-TT, rs1799794-GG with rs861530-AG, rs1799794-AA with rs709399-AA, rs1799794-GG with rs709399-AG, rs1799796-AG with rs861539-TT, rs1799796-AG with rs709399-AA, rs1799796-GG with rs709399-AA, rs861539-TT with rs861530-GG, rs861539-CC with rs709399-AA, rs861539CT with rs709399-GG, rs861539-TT with rs709399-AA and rs861530- AG with rs709399AA) are associated with an increased thyroid cancer risk. The present combined genotype frequency and their significance in thyroid carcinogenesis may show the synergistic effect of the selected polymorphisms on the said disease. So for only a few studies have been conducted regarding the combined genotypes of XRCC3 SNPs and their involvement in cancer, $[59,20]$ but the results are not comparable because they genotyped different SNPs.

Before making any conclusion, several potential limitations of this study should be addressed. First of all future studies, should incorporate a large sample size with various ethnics to confirm the association between XRCC3 SNPs and thyroid carcinogenesis. Secondly, few well known risk factors for thyroid cancer have been discovered, therefore other genetic and environmental factors should be assessed. Thirdly, subjects in this case control study came from two hospitals and this may cause selection bias that could have substantial impact on overall conclusions. As a result, large scale studies that adjusting for a wide range of factors should be recommended to validate our findings.

The current available data suggest that the identification of patterns of genetic variations, in the form of haplotypes rather than single or point variations, may present a more promising approach to predict a person's response to drug therapy, and allow for highly individualized treatment of patients with complex diseases, such as cancer and their associated risk factors. In conclusion, our findings suggest a potential association between XRCC3 (rs1799794, rs1799794, rs861539, rs861530 and rs709399) genetic variants and thyroid cancer disease susceptibility. Nevertheless, these conclusions should be re-confirmed with large samples and various ethnicities.

\section{KARGER}




\section{Cellular Physiology Cell Physiol Biochem 2017;42:22-33

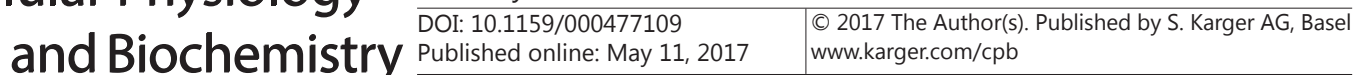

Sarwar et al.: XRCC3 Polymorphisms and Thyroid Cancer

\section{Acknowledgements}

We wish to thank all staff and patients of Nuclear Medicine Oncology \& Radiotherapy Institute (NORI), Islamabad, and volunteers who participated in this study. We acknowledge financial and infrastructural help from Higher Education Commission of Pakistan (HEC) and COMSATS Institute of Information Technology (CIIT), Islamabad.

\section{Disclosure Statement}

The authors declare that they have no competing interests.

\section{References}

1 Bukhari U, Sadiq S, Memon J, Baig F: Thyroid carcinoma in Pakistan: a retrospective review of 998 cases from an academic referral center. Hematol Oncol Stem Cell Ther 2009;2:345-348.

- Pellegriti G, Frasca F, Regalbuto C, Squatrito S, Vigneri R: Worldwide increasing incidence of thyroid cancer: update on epidemiology and risk factors. J Cancer Epidemiol 2013;2013:965212.

- 3 Bukhari MH, Niazi S, Hanif G, Qureshi SS, Munir M, Hasan M, Naeem S: An updated audit of fine needle aspiration cytology procedure of solitary thyroid nodule. Diagn Cytopathol 2008;36:104-112.

-4 Smailyte G, Miseikyte-Kaubriene E, Kurtinaitis J, Sakoda L, Horn-Ross P, Feldt-Rasmussen U: Increasing thyroid cancer incidence in Lithuania in 1978-2003. BMC Cancer 2006;6:284.

5 Kilfoy BA, Zheng T, Holford TR, Han X, Ward MH, Sjodin A, Zhang Y, Bai Y, Zhu C, Guo GL, Rothman N: International patterns and trends in thyroid cancer incidence, 1973-2002. Cancer Causes Control 2009;20:525-531.

6 Shaw A, Semenciw R, Mery L: Cancer in Canada Fact Sheet Series\# 1: Thyroid cancer in Canada. Chronic Dis Inj Can 2014;34:64-68.

7 Brown P, Jiang H, Ezzat S, Sawka AM, Guay B, Johnson-Obaseki S: A detailed spatial analysis on contrasting cancer incidence patterns in thyroid and lung cancer in Toronto women. BMC Public Health 2016;16:950.

-8 McNally RJQ Blakey K, James PW, Gomez Pozo B, Basta NO, Hale J: Increasing incidence of thyroid cancer in Great Britain, 1976-2005: age-period-cohort analysis. Eur J Epidemiol 2012;27:615-622.

-9 Giri S, Pathak R, Aryal MR, Karmacharya P, Bhatt VR, Martin MG: Seasonal variation in the presentation of thyroid cancer in the USA: an analysis of the Surveillance, Epidemiology and End Results Registry. Cancer Causes Control 2014;25:1583.

10 Nikiforov YE: Is ionizing radiation responsible for the increasing incidence of thyroid cancer? Cancer 2010;116:1626-1628.

-11 Ferguson LR, Chen H, Collins AR, Connell M, Damia G, Dasgupta S, Malhotra M, Meeker AK, Amedei A, Amin A, Ashraf SS: Genomic instability in human cancer: Molecular insights and opportunities for therapeutic attack and prevention through diet and nutrition. Semin Cancer Biol 2015;35:S5-S24.

12 Jeggo PA, Pearl LH, Carr AM: DNA repair, genome stability and cancer: a historical perspective. Nat Rev Cancer 2015;16:35-42.

13 Kavanagh JN, Redmond KM, Schettino G, Prise KM: DNA Double Strand Break Repair: A Radiation Perspective. Antioxid Redox Signal 2013;18:2458-2472.

14 Takata M, Sasaki MS, Sonoda E, Morrison C, Hashimoto M, Utsumi H, Yamaguchi-Iwai Y, Shinohara A, Takeda S: Homologous recombination and non-homologous end-joining pathways of DNA double-strand break repair have overlapping roles in the maintenance of chromosomal integrity in vertebrate cells. EMBO J 1998;17:5497-5508.

15 Rowe JM, Kim HT, Cassileth PA, Lazarus HM, Litzow MR, Wiernik PH, Tallman MS: Adult patients with acute myeloid leukemia who achieve complete remission after 1 or 2 cycles of induction have a similar prognosis. Cancer 2010;116:5012-5021.

16 Kurumizaka H, Ikawa S, Nakada M, Eda K, Kagawa W, Takata M, Takeda S, Yokoyama S, Shibata T: Homologouspairing activity of the human DNA-repair proteins XRCC3*Rad51C. Proc Natl Acad Sci USA 2001;98:55385543. 


\section{Cellular Physiology Cell Physiol Biochem 2017;42:22-33 and Biochemistry Published onIme: Vay 11, $2017 \quad \begin{aligned} & \text { DOI: 10.1159/000477109 } 2017 \text { The Author(s). Published by S. Karger AG, Basel } \\ & \text { www.karger.com/cpb }\end{aligned}$}

Sarwar et al.: XRCC3 Polymorphisms and Thyroid Cancer

17 Brenneman MA, Wagener BM, Miller CA, Allen C, Nickoloff JA: XRCC3 controls the fidelity of homologous recombination: roles for XRCC3 in late stages of recombination. Mol Cell 2002;10:387-95.

18 Zhan P, Wang Q, Qian Q Yu L-K: XRCC3 Thr241Met gene polymorphisms and lung cancer risk: a metaanalysis. J Exp Clin Cancer Res 2013;32:1.

-19 Auranen A, Song H, Waterfall C, DiCioccio RA, Kuschel B, Kjaer SK, Hogdall E, Hogdall C, Stratton J, Whittemore AS, Easton D: Polymorphisms in DNA repair genes and epithelial ovarian cancer risk. Int J Cancer 2005;117:611-618.

20 Yuan C, Liu X, Yan S, Wang C, Kong B: Analyzing association of the XRCC3 gene polymorphism with ovarian cancer risk. Biomed Res Int 2014;2014:648137.

21 Guo S, Li X, Gao M, Li Y, Song B, Niu W: The Relationship between XRCC1 and XRCC3 Gene Polymorphisms and Lung Cancer Risk in Northeastern Chinese. PLoS One 2013;8:e56213.

22 Tian X, Tian Y, Ma P, Sui C, Meng F, Li Y, Fu L, Jiang T, Wang Y, Jiang Y: Association between the XRCC3 C241T polymorphism and lung cancer risk in the Asian population. Tumor Biol 2013;34:2589-2597.

23 He XF, Wei W, Su J, Yang Z-X, Liu Y, Zhang Y, Ding DP, Wang W: Association between the XRCC3 polymorphisms and breast cancer risk: meta-analysis based on case-control studies. Mol Biol Rep 2012;39:5125-5134.

24 Cheng C-X, Xue M, Li K, Li W-S: Predictive Value of XRCC1 and XRCC3 Gene Polymorphisms for Risk of Ovarian Cancer Death After Chemotherapy. Asian Pacific J Cancer Prev 2012;13:2541-2545.

-25 Su Y, Zhang H, Xu F, Kong J, Yu H, Qian B: DNA repair gene polymorphisms in relation to non-small cell lung cancer survival. Cell Physiol Biochem 2015;36:1419-1429.

-26 Sarwar R, Bashir K, Saeed S, Mahjabeen I, Kayani MA: Association of Promoter Polymorphisms in Xrcc2 Gene Involved in DNA Double Strand Break Repair and Increased Susceptibility to Thyroid Cancer Risk in Pakistani Population. J Carcinog Mutagen 2016;7:3.

-27 Yang C, Wang Q Liu X, Cheng X, Jiang X, Zhang Y, Feng Z, Zhou P: NU7441 Enhances the Radiosensitivity of Liver Cancer Cells. Cell Physiol Biochem 2016;38:1897-1905.

-28 Skeeles LE., Fleming JL, Mahler KL,Toland, AE: The impact of 3' UTR variants on differential expression of candidate cancer susceptibility genes. PloS One 2013;8:e58609.

29 Landi D, Gemignani F, Pardini B, Naccarati A, Garritano S, Vodicka P, Vodickova L, Canzian F, Novotny J, Barale R, Landi S: Identification of candidate genes carrying polymorphisms associated with the risk of colorectal cancer by analyzing the colorectal mutome and microRNAome. Cancer 2012;118:4670-4680.

-30 Arnold M, Ellwanger DC, Hartsperger ML, Pfeufer A, Stümpflen V: Cis-acting polymorphisms affect complex traits through modifications of microRNA regulation pathways. PLoS One 2012;7:e36694.

31 Shen MR, Jones IM, Mohrenweiser H: Nonconservative Amino Acid Substitution Variants Exist at Polymorphic Frequency in DNA Repair Genes in Healthy Humans. Cancer Res 1998;58:604-608.

-32 Matullo G, Palli D, Peluso M, Guarrera S, Carturan S, Celentano E, Krogh V, Munnia A, Tumino R, Polidoro S, Piazza A: XRCC1, XRCC3, XPD gene polymorphisms, smoking and (32)P-DNA adducts in a sample of healthy subjects. Carcinogenesis 2001;22:1437-1445.

-33 Lindh AR, Rafii S, Schultz N, Cox A, Helleday T: Mitotic defects in XRCC3 variants T241M and D213N and their relation to cancer susceptibility. Hum Mol Genet 2006;15:1217-1224.

-34 Manuguerra M, Saletta F, Karagas MR, Berwick M, Veglia F, Vineis P, Matullo G: XRCC3 and XPD/ERCC2 single nucleotide polymorphisms and the risk of cancer: a HuGE review. Am J Epidemiol 2006;164:297302.

-35 Werbrouck J, De Ruyck K, Duprez F, Veldeman L, Claes K, Van Eijkeren M, Boterberg T, Willems P, Vral A, De Neve W, Thierens H: Acute normal tissue reactions in head-and-neck cancer patients treated with IMRT: influence of dose and association with genetic polymorphisms in DNA DSB repair genes. Int J Radiat Oncol Biol Phys 2009;73:1187-1195.

-36 Sturgis EM, Zhao C, Zheng R, Wei Q: Radiation Response Genotype and Risk of Differentiated Thyroid Cancer: A Case-Control Analysis. Laryngoscope 2005;115:938-945.

37 Bastos HN, Antão MR, Silva SN, Azevedo AP, Manita I, Teixeira V, Pina JE, Gil OM, Ferreira TC, Limbert E, Rueff J: Association of Polymorphisms in Genes of the Homologous Recombination DNA Repair Pathway and Thyroid Cancer Risk. Thyroid 2009;19:1067-1075.

-38 Fayaz S, Karimmirza M, Tanhaei S, Fathi M, Torbati PM, Fard-Esfahani P: Increased Risk of Differentiated Thyroid Carcinoma with Combined Effects of Homologous Recombination Repair Gene Polymorphisms in an Iranian Population. Asian Pacific J Cancer Prev 2013;14:6727-6731. 


\section{Cellular Physiology Cell Physiol Biochem 2017;42:22-33 and Biochemistry Published ontune: May 11, $2017 \quad \begin{aligned} & \text { DOI: 10.1159/000477109 } 2017 \text { The Author(s). Published by S. Karger AG, Basel } \\ & \text { www.karger.com/cpb }\end{aligned}$}

Sarwar et al.: XRCC3 Polymorphisms and Thyroid Cancer

-39 Yu XL, Liu H, Wang B, Fu Z-J, Yuan Y, Yan S-L, Zhao WJ, Wang YG, Cai J: Significant associations between $\mathrm{X}$-ray repair cross-complementing group 3 genetic polymorphisms and thyroid cancer risk. Tumor Biol 2014;35:2009-2015.

40 Yuan K, Huo M, Sun Y, Wu H, Chen H, Wang Y, Fu R.: Association between X-ray repair cross-complementing group 3 (XRCC3) genetic polymorphisms and papillary thyroid cancer susceptibility in a Chinese Han population. Tumor Biol 2016;37:979-987.

-41 Siraj AK, Al-Rasheed M, Ibrahim M, Siddiqui K, Al-Dayel F, Al-Sanea O, Uddin S, Al-Kuraya K: RAD52 polymorphisms contribute to the development of papillary thyroid cancer susceptibility in Middle Eastern population. J Endocrinol Invest 2008;31:893-899.

42 Akulevich NM, Saenko VA, Rogounovitch TI, Drozd VM, Lushnikov EF, Ivanov VK, Mitsutake N, Kominami R, Yamashita S: Polymorphisms of DNA damage response genes in radiation-related and sporadic papillary thyroid carcinoma. Endocr Relat Cancer 2009;16:491-503.

-43 Krupa R, Blasiak J: An association of polymorphism of DNA repair genes XRCC1 and XRCC3 with colorectal cancer. J Exp Clin Cancer Res 2004;23:285-94.

44 Jin MJ, Chen K, Song L, Fan CH, Chen Q Zhu YM, Ma XY, Yao KY: The association of the DNA repair gene XRCC3 Thr241 Met polymorphism with susceptibility to colorectal cancer in a Chinese population. Cancer Genet Cytogenet 2005;163:38-43.

45 Zhou K, Liu Y, Zhang H, Liu H, Fan W, Zhong Y, Xu Z, Jin L, Wei Q, Huang F, Lu D: XRCC3 haplotypes and risk of gliomas in a Chinese population: A hospital-based case-control study. Int J Cancer 2009;124:2948-2953.

46 Mittal RD, Mandal RK, Gangwar R: Base excision repair pathway genes polymorphism in prostate and bladder cancer risk in North Indian population. Mech Ageing Dev 2012;133:127-32.

47 Hamdy MS, El-Haddad AM, Bahaa El-Din NM, Makhlouf MM, Abdel-Hamid SM: RAD51 and XRCC3 Gene Polymorphisms and the Risk of Developing Acute Myeloid Leukemia. J Investig Med 2011;59:1124-1130.

-48 Seedhouse C, Faulkner R, Ashraf N, Das-Gupta E, Russell N: Polymorphisms in Genes Involved in Homologous Recombination Repair Interact to Increase the Risk of Developing Acute Myeloid Leukemia. Clin Cancer Res 2004;10:2675-2680.

49 Xu F, Li D, Zhang Q Fu Z, Yuan W, Pang D, Li D: Association of CD27 and CD70 gene polymorphisms with risk of sporadic breast cancer in Chinese women in Heilongjiang Province. Breast Cancer Res Treat 2012;133:1105-1113.

50 Han S, Zhang H-T, Wang Z, Xie Y, Tang R, Mao Y, Li Y: DNA repair gene XRCC3 polymorphisms and cancer risk: a meta-analysis of 48 case-control studies. Eur J Hum Genet 2006;14:1136-1144.

51 Matullo G, Guarrera S, Sacerdote C, Polidoro S, Davico L, Gamberini S, Karagas M, Casetta G, Rolle L, Piazza A, Vineis P: Polymorphisms/Haplotypes in DNA Repair Genes and Smoking: A Bladder Cancer Case-Control Study. Cancer Epidemiol Biomarkers Prev 2005;14:2569-2578.

52 Peng Q, Mo C, Tang W, Chen Z, Li R, Zhai L, Yang S, Wu J, Sui J, Li S, Qin X: DNA repair gene XRCC3 polymorphisms and bladder cancer risk: a meta-analysis. Tumor Biol 2014;35:1933-1944.

53 Ni HX, Bian JC, Shen Q Tang HW, Zhu QX, Wu Y: Genetic polymorphisms of XRCC3 and susceptibility of papillary thyroid carcinoma. Fudan Univ J Med Sci 2006;33:147-152.

54 Luo HC, Zhang H-B, Xin XJ, Huang WX: Haplotype-based case-control study of DNA repair gene XRCC3 and hepatocellular carcinoma risk in a Chinese population. Tumor Biol 2014;35:3415-3419.

-55 García-Closas M, Egan KM, Newcomb PA, Brinton LA, Titus-Ernstoff L, Chanock S, Welch R, Lissowska J, Peplonska B, Szeszenia-Dabrowska N, Zatonski W: Polymorphisms in DNA double-strand break repair genes and risk of breast cancer: two population-based studies in USA and Poland, and meta-analyses. Hum Genet 2006;119:376-388.

56 Kuschel B, Auranen A, McBride S, Novik KL, Antoniou A, Lipscombe JM, Day NE, Easton DF, Ponder BA, Pharoah PD, Dunning A: Variants in DNA double-strand break repair genes and breast cancer susceptibility. Hum Mol Genet 2002;11:1399-1407.

57 Han J, Colditz GA, Samson LD, Hunter DJ: Polymorphisms in DNA double-strand break repair genes and skin cancer risk. Cancer Res 2004;64:3009-3013.

58 Smedby KE, Lindgren CM, Hjalgrim H, Humphreys K, Schöllkopf C, Chang ET, Roos G, Ryder LP, Falk KI, Palmgren J, Kere J: Variation in DNA repair genes ERCC2, XRCC1, and XRCC3 and risk of follicular lymphoma. Cancer Epidemiol Biomarkers Prev 2006;15:258-265.

59 Yan L, Li Q, Li X, Ji H, Zhang L: Association Studies Between XRCC1, XRCC2, XRCC3 Polymorphisms and Differentiated Thyroid Carcinoma. Cell Physiol Biochem 2016;38:1075-1084. 